TITLE:

\title{
Market power and trading strategies on the electricity market: A market design view
}

$\operatorname{AUTHOR}(\mathrm{S})$ :

Reinisch, W; Tezuka, T

\section{CITATION:}

Reinisch, W ... [et al]. Market power and trading strategies on the electricity market: A market design view. IEEE TRANSACTIONS ON POWER SYSTEMS 2006, 21(3): 1180-1190

\section{ISSUE DATE:}

2006-08

URL:

http://hdl.handle.net/2433/50252

\section{RIGHT:}

(c)2006 IEEE. Personal use of this material is permitted. However, permission to reprint/republish this material for advertising or promotional purposes or for creating new collective works for resale or redistribution to servers or lists, or to reuse any copyrighted component of this work in other works must be obtained from the IEEE. 


\title{
Market Power and Trading Strategies on the Electricity Market: A Market Design View
}

\author{
Walter Reinisch and Tetsuo Tezuka
}

\begin{abstract}
In this paper, we examine auction-based electricity markets from the viewpoint of a power company in order to evaluate the market design. Optimal trading strategies under uncertainty are developed by abolishing the widespread assumption that a single company has no influence on the market price. The resulting trading strategies explain the high prices and the high volatility observed in real electricity auction markets and show to what extent power companies can manipulate the market price. From these trading strategies, we derive critical points in market design and formulate necessary and sufficient conditions that characterize a competitive electricity auction market. Unfortunately, these conditions are far from being realizable in practice, from which follows that electricity auction markets cannot be competitive.
\end{abstract}

Index Terms-Competition, double-sided auctions, electricity market, market design, market power, oligopoly, trading strategies, uniform-price auctions.

\section{INTRODUCTION}

$\mathbf{W}$ ITH the deregulation of electricity markets in the last years, the electric power industry now faces a situation of competition. Although the introduction of competition was believed to result in lower electricity prices and more efficient production, many countries now experience electricity market prices higher than expected by economic considerations.

The reason for these market inefficiencies is, in common sense of literature, market power and insufficient competition: big companies can influence the market price by means of their strategy. On top of that, even small suppliers seem to be able to exercise market power in times of steep supply function [1], and suppliers do not have any incentive to bid at marginal cost [2]. To resolve these problems, some changes have been applied to the electricity markets, and different approaches have been discussed in research work. There are basically four solution paths. In order to increase competition, one solution is to increase the number of market participants by dividing big companies into smaller ones, split capacity from big companies, or wait for new entry [3].

Second, a change in the auction structure may result in less chance to exercise market power [4]. Ren and Galiana [5], [6] compare the two auction structures pay-as-bid and uniform-price and conclude that pay-as-bid has some statistical advantages over uniform-price auctions. They raise the question

Manuscript received September 22, 2005; revised February 9, 2006. Paper no. TPWRS-00599-2005.

The authors are with the Graduate School of Energy Science, Kyoto University, Kyoto 606-8501, Japan.

Digital Object Identifier 10.1109/TPWRS.2006.876675 whether there will be a difference in the possibility of gaming to the participants.

A third way is to introduce demand-side management to decrease market price by changing the demand curve. Kian et al. [4] simulated the electricity market assuming a Nash-Cournot game and that the companies' profit function coefficients are normally distributed and publicly available. The conclusion is that double-sided auctions are preferable because of price responsive demand.

Finally, as a last resort, the introduction of price caps may try to forcibly keep the prices low. However, this approach failed in the California electricity market [1], [7] and may lead to reliability problems [8].

In order to explain the high market prices, we may consult game theory, which knows four different situations between competitors [9]: cooperative and noncooperative games, both of which being with conflict or without conflict. Since cooperation is illegal, there are two cases left to consider.

In a noncooperative game with conflict, which is the basic assumption in all the literature on market power, a company is considered being in conflict with competing companies when deciding the bidding price because the competitor's strategy is unknown. Due to this assumption of a certain amount of competition, the only possibility to achieve higher market prices lies in utilizing an advantage in competition due to excess information or higher generation capacity. This leads to the discussion of market power [4] and to new trading strategies that utilize market information to achieve higher revenues [10], [11].

In a noncooperative game without conflict, companies may influence the market price without communication. They may bid at a price higher than marginal cost without considering the competitors' strategies. If a market turns out to fall in this category of games, it is not competitive, and changes in market structure are necessary. Since we cannot exclude the possibility of a game without conflict, we investigate what conditions have to be fulfilled to achieve a state where the optimal bidding price is equal to marginal cost. In this optimal state of the market, a company does not have influence on the market price any more. Therefore, the resulting conditions also define a market of perfect competition.

We estimate the optimal bidding price on an electricity auction market from the viewpoint of a company of arbitrary size by maximizing the expected profit of the trading decision for one auction, considering that the company has influence on the market price only by withholding generation capacity. We assume that the market price arises from the intersection of supply and demand function and that the market price may be considered as a random variable with arbitrary probability 
density function. No further assumptions are needed for our investigation.

The resulting trading strategies are then analyzed from the viewpoint of market design in order to find conditions that annihilate the company's influence on the market price.

\section{Optimal TRAding STRATEgIES UNDER UNCERTAINTY}

The following trading strategies optimize a company's total profit, say, $r$, dependent on the market price, which we denote with $p$. Because the market price is uncertain, it is considered as a random variable, say, $P . P$ is defined by its probability density function $\mathcal{G}_{P}(p)$, and $p$ is therefore a sample of $P$. We further denote the cumulative distribution function of $P$ with $\Gamma_{P}(x), \Gamma_{P}(x)=\int_{-\infty}^{x} \mathcal{G}_{P}(p) d p$ and $\int_{-\infty}^{\infty} \mathcal{G}_{P}(p) d p=1$.

The company under consideration has a total generation capacity of $Q$ and makes $N$ bids on the auction market, which we identify by the index $i, 1 \leq i \leq N$. We denote the bids with $b_{i}$, the achieved price for a bid with $p_{i}$, and the volume of the bids with $q_{i}$, for which holds $\sum_{i=1}^{N} q_{i}=Q$. The marginal cost for production of the quantity $q_{i}$ is denoted with $c_{i}$. The number of bids is chosen equal to the number of power plants, i.e., one bid for every power plant, so $q_{i}$ is equal to the capacity of power plant $i$, and $c_{i}$ is equal to its marginal cost. The index $i$ is chosen such that the bids' cost are ordered

$$
c_{1} \leq c_{2} \leq \cdots \leq c_{N}
$$

and therefore the corresponding bids are ordered as well

$$
b_{1} \leq b_{2} \leq \cdots \leq b_{N}
$$

The total profit $r$ over all power plants is the sum over the plants' profits $r_{i}$

$$
r=\sum_{i=1}^{N} r_{i}
$$

and the power plant's profit $r_{i}$ is revenue less cost

$$
r_{i}=\left(p_{i}-c_{i}\right) q_{i} .
$$

The relation between the market price $p$ and the power plant's achieved price $p_{i}$ depends on the auction format, so we will define this relation in the sections of the respective auctions.

The expected profit $\bar{r}$ is

$$
\bar{r}=\int_{-\infty}^{\infty} \mathcal{G}_{P}(p) r d p
$$

which is dependent on the bids $b_{i}$. The optimal trading strategy is therefore the set of bids $\left\{b_{1}, \ldots, b_{N}\right\}$ that maximizes (5).

\section{A. Optimal Trading Strategy for the Generation-Only Company}

The company we consider in this section does not have access to the retail market. All capacity is offered on the electricity spot market.

To derive the optimal trading strategy, we calculate the maximum expected profit for the two cases of pay-as-bid and uniform-price auctions.

1) Pay-as-Bid Auctions: In pay-as-bid auctions, the achieved price of a power plant $p_{i}$ is equal to the bidding price $b_{i}$ if the corresponding bid wins the auction, i.e., if the bid $b_{i}$ is smaller than or equal to the market price $p$. The market price $p$ is the price of the most expensive power plant that wins the auction. The revenue of a single power plant is therefore, with (4)

$$
r_{i}= \begin{cases}\left(b_{i}-c_{i}\right) q_{i}, & \text { for } b_{i} \leq p \\ 0, & \text { for } b_{i}>p .\end{cases}
$$

For this calculation of the expected profit, we assume a perfect competitive market, so that the company does not have influence on the market price. Considering the uncertainty of the market price, the expected profit $\bar{r}_{i}$ is $\bar{r}_{i}=\int_{-\infty}^{\infty} \mathcal{G}_{P}(p) r_{i} d p$, which gives, with (6)

$$
\bar{r}_{i}=\int_{b_{i}}^{\infty} \mathcal{G}_{P}(p)\left(b_{i}-c_{i}\right) q_{i} d p
$$

and, since $\left(b_{i}-c_{i}\right) q_{i}$ is independent of $p$

$$
\bar{r}_{i}=\left(1-\Gamma_{P}\left(b_{i}\right)\right)\left(b_{i}-c_{i}\right) q_{i} .
$$

The first-order condition to maximize $\bar{r}_{i}$ for $b_{i},\left(d \bar{r}_{i}\right) /\left(d b_{i}\right)=0$ yields

$$
1-\Gamma_{P}\left(b_{i}\right)-\left(b_{i}-c_{i}\right) \mathcal{G}_{P}\left(b_{i}\right)=0
$$

which may be solved numerically for given $\operatorname{cost} c_{i}$ under the assumption of a specific distribution.

In order to evaluate the potential of bidding at higher prices than marginal cost, we have to examine the probability distribution in more detail. There are two cases to consider: a distribution with a finite maximum price, say, $p^{\max }$, and an unbounded distribution. In the latter case

$$
b_{i}>c_{i}
$$

holds for the solution of (9), independent of $c_{i}$, since $1-\Gamma_{P}\left(b_{i}\right)>0$ and $\mathcal{G}_{P}\left(b_{i}\right)>0$. In the former case, $b_{i}>c_{i}$ if $c_{i}$ is less than the distribution's upper bound.

Under normal equilibrium conditions, i.e., $c_{i}<p^{\max }$, and, since we assumed that the company cannot influence the market price, in a perfect competitive environment, the optimal bid is higher than marginal cost. This characteristic of the pay-as-bid auction would give unsatisfying high prices on the electricity 
market, and therefore, it would not be our preferred choice in market design.

2) Uniform-Price Auction: In the case of a uniform-price auction, the achieved prices $p_{i}$ of the power plants are equal to the uniform market price $p, p=p_{1}=p_{2}=\cdots=p_{N}$.

We begin the calculation of the optimal strategy with the case of a perfect competitive environment. This assumption will be abandoned later on.

a) Company Is a Price Taker: In case the company does not have influence on the market price, the total profit $r$ is

$$
r(p)= \begin{cases}0, & \text { for } p<b_{1} \\ p \sum_{i=1}^{n} q_{i}-\sum_{i=1}^{n} q_{i} c_{i}, & \text { for } b_{n} \leq p<b_{n+1} \\ p \sum_{i=1}^{N} q_{i}-\sum_{i=1}^{N} q_{i} c_{i}, & \text { for } b_{N} \leq p .\end{cases}
$$

$n$ is the index of the bid under consideration, $1 \leq n<N$. The expected profit is

$$
\begin{aligned}
\bar{r}=\sum_{n=1}^{N-1} \int_{b_{n}}^{b_{n+1}} & \mathcal{G}_{P}(p)\left(p \sum_{i=1}^{n} q_{i}-\sum_{i=1}^{n} q_{i} c_{i}\right) d p \\
& +\int_{b_{N}}^{\infty} \mathcal{G}_{P}(p)\left(p \sum_{i=1}^{N} q_{i}-\sum_{i=1}^{N} q_{i} c_{i}\right) d p
\end{aligned}
$$

With the first-order condition $(d \bar{r}) /\left(d b_{n}\right)=0$ for $1 \leq n \leq N$, the optimal bids are $b_{n}=c_{n}$ for all $N$ bids (see the Appendix for the derivation). This corresponds to the widespread economic understanding that led to the deregulation process in the electricity industry.

b) Company Does Influence the Market Price: The power company may try to influence the market price by adapting the bid of a single power plant. If the company succeeds, it loses the bid for this power plant but has in return a higher revenue on the remaining power plants. This strategy can be accomplished without having too much risk, if the bids of the remaining power plants are reasonably low.

Therefore, by withholding some generation capacity, a generation company will try to influence the market's supply function and increase the resulting market price. However, rather than shutting down the power plant, the generation company may decide to bid above the expected market price. This increases the chance of selling additional electricity in case the market price is even higher than expected. Fig. 1 illustrates this strategy: bidding a cheap power plant (top graph in Fig. 1) at a higher price (bottom graph in Fig. 1) does increase the market price.

This means that we have to drop the assumption that the company does not have influence on the market price, which was the condition for the above calculation that led to an optimal $b_{n}$ equal to $c_{n}$. In order to formulate this situation, we split the market price into two components: $\tilde{p}$ denotes the price without influence of the company, and the resulting market price is denoted with $\hat{p}$. Note that, even though $\tilde{p}$ is a price without influence of the company, $\tilde{p}$ is not a competitive price, because the company is withholding generation capacity.

As demonstrated before, the company may influence the market price by withholding some capacity. If we denote this capacity with $\Delta q$, the influenced market price $\hat{p}$ is, without
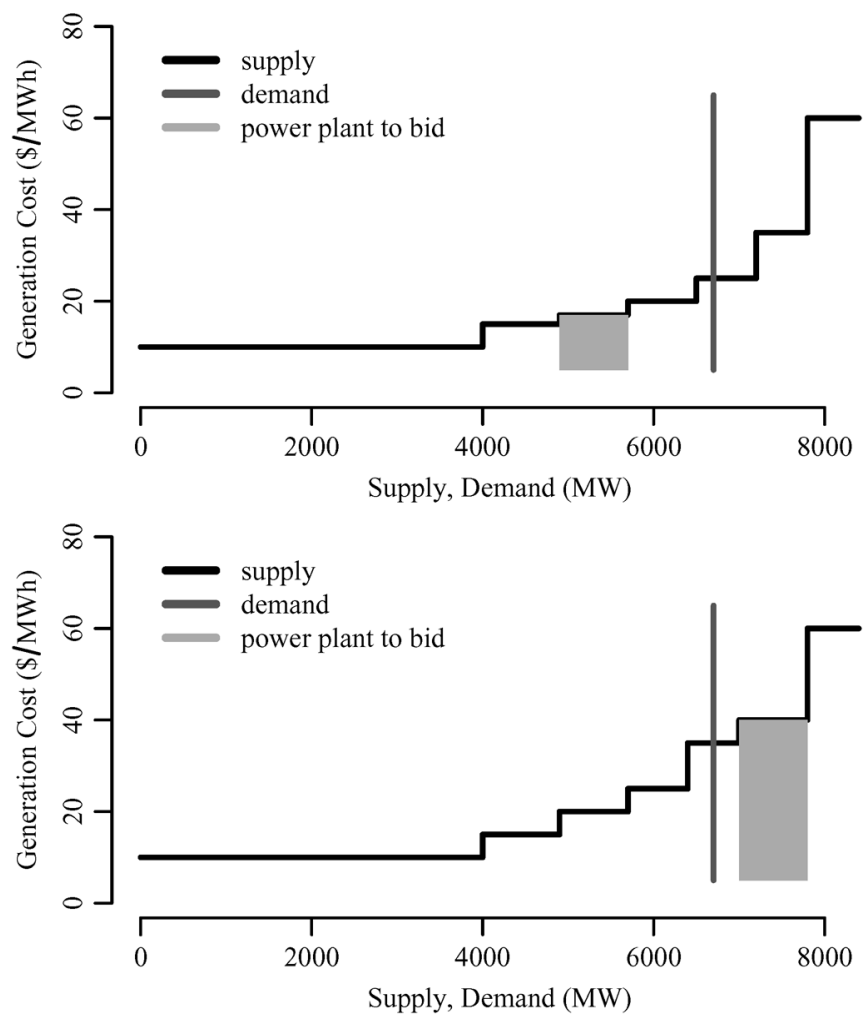

Fig. 1. Merit order cost curve of a small power system depending on the bid of a single power plant at marginal cost (top) or at a price higher than the market price (bottom).

loss of generality, a function of $\Delta q, \hat{p}=f(\Delta q, \mathbf{a}) \cdot f(\Delta q, \mathbf{a})$ describes the electricity market in all its complexity, including competitors and physical constraints. a is an arbitrary vector that represents the state of the market. This function has two basic characteristics: if the company does not withhold some capacity $\Delta q=0$, then there is no change in market price $\hat{p}=\tilde{p}$, and therefore, $f(0, \mathbf{a})=\tilde{p}$. Furthermore, $f(\Delta q, \mathbf{a})$ must rise as $\Delta q$ rises, so $(\partial f(\Delta q, \mathbf{a})) /(\partial \Delta q) \geq 0$. In case $(\partial f(\Delta q, \mathbf{a})) /(\partial \Delta q)=0$ for all $\Delta q$, then the market is perfectly competitive.

These characteristics of $f(\Delta q, \mathbf{a})$ allow for a linear approximation in the point $\Delta q=0$. With the slope of $f(\Delta q, \mathbf{a})$ in $\Delta q=$ $0,(\partial f(\Delta q, \mathbf{a})) /\left.(\partial \Delta q)\right|_{\Delta q=0}=k$, we get the approximation ${ }^{1}$

$$
\hat{p}=\tilde{p}+k \Delta q
$$

$k$ quantifies the possible influence of a single company on the resulting market price. We will discuss $k$ in more detail later on in this paper.

The new market price $\hat{p}$ results from different optimal bids. If we split the bids in the same manner into a bid without price influence denoted by $\tilde{b}_{n}$ and the company's price influence, this price influence must be equal to $k \Delta q_{n}$, in order to achieve the change in price $\hat{p}-\tilde{p}=k \Delta q . \Delta q_{n}$ denotes the capacity that

${ }^{1}$ We assumed that $f(\Delta q, \mathbf{a})$ is differentiable in $\Delta q$. For non-differentiable $f(\Delta q, \mathbf{a})$, we may replace the differentiation with the differential quotient to achieve the same approximation, i.e., $k=(f(\Delta q+h, \mathbf{a})-f(\Delta q-$ $h, \mathbf{a})) /(2 h)$, for an arbitrary $h>0$. The differentiations in the description of $f(\Delta q, \mathbf{a})$ then change correspondingly. 
the company withholds if the corresponding bid is the highest winning bid of the company. The new influenced bids, denoted with $\hat{b}_{n}$, are therefore $\hat{b}_{n}=\tilde{b}_{n}+k \Delta q_{n}$. The right graph of Fig. 2 shows the relationship between the bids, the prices, and the withheld capacity. For simplicity, the graphs are built up of smooth bidding functions $b(q), \tilde{b}(q)$, and $\hat{b}(q)$ instead of discrete bids $b_{n}, \tilde{b}_{n}$ and $\hat{b}_{n}$. The functions $s(q), d(q)$ and $l(q)$ are described in detail in Section II-C1.

Considering the situation that $\hat{b}_{n} \leq \hat{p}<\hat{b}_{n+1}$, the power plants with bids $\hat{b}_{n+1}$ and higher are too expensive, lose the auction, and may influence the market price. According to above consideration, only those power plants contribute to $\Delta q_{n}$, which could have won the auction under uninfluenced conditions. In other words, those power plants that fulfill the conditions $\tilde{b}_{n}<\tilde{p}$ and $\hat{b}_{n}>\hat{p}$ are responsible for the change in the market price. To formulate this relation, we define the step-function $\mathrm{g}(x)$

$$
g(x)= \begin{cases}0, & \text { for } x<0 \\ 1, & \text { for } x \geq 0\end{cases}
$$

The quantity $\Delta q_{n}$ effecting the market price then results in

$$
\Delta q_{n}=\sum_{i=n+1}^{N} \mathrm{~g}\left(\tilde{p}-c_{i}\right) q_{i}
$$

for $1 \leq n<N$. In case the resulting market price is greater than the bid of the most expensive power plant $\hat{b}_{N}$, then $\Delta q=0$ and therefore $\hat{b}_{N}=\tilde{b}_{N}$ and also $\hat{p}=\tilde{p}$. We can therefore write for $\hat{b}_{n}$

$$
\begin{aligned}
& \qquad \hat{b}_{n}= \begin{cases}\tilde{b}_{n}+k \sum_{i=n+1}^{N} \mathrm{~g}\left(\tilde{b}-c_{i}\right) q_{i}, & \text { for } 1 \leq n<N \\
\tilde{b}_{n}, & \text { for } n=N\end{cases} \\
& \text { and for } \hat{p} \\
& \qquad \hat{p}= \begin{cases}\tilde{p}+k \sum_{i=n+1}^{N} \mathrm{~g}\left(\tilde{p}-c_{i}\right) q_{i}, & \text { if } 1 \leq n<N \\
\tilde{p}, & \text { if } n=N .\end{cases}
\end{aligned}
$$

Since $k$ is not exactly known to the company, we assume $k$ being a sample from a random variable $K$ with an arbitrary probability density function $\mathcal{G}_{K}(k)$. If we compare $\tilde{p}$ with $p$, we find that they have equal distribution, since the corresponding bids differ in volume, not in price. So $\tilde{p}$ is a sample of $P$. Considering the random variables $P$ and $K$ being independent, the expected profit $\bar{r}$ is

$$
\bar{r}=\int_{-\infty}^{\infty} \int_{-\infty}^{\infty} \mathcal{G}_{K}(k) \mathcal{G}_{P}(\tilde{p}) r(\tilde{p}, k) d \tilde{p} d k .
$$

By rewriting the profit (11) for $\hat{p}$ and inserting (17), we get

$$
\begin{aligned}
\bar{r}= & \int_{-\infty}^{\infty} \mathcal{G}_{K}(k)\left(\sum_{n=1}^{N-1} \int_{\tilde{b}_{n}}^{\tilde{b}_{n+1}} \mathcal{G}_{P}(\tilde{p})\right. \\
& \times\left(\left(\tilde{p}+k \sum_{i=n+1}^{N} \mathrm{~g}\left(\tilde{p}-c_{i}\right) q_{i}\right) \sum_{i=1}^{n} q_{i}-\sum_{i=1}^{n} q_{i} c_{i}\right) d \tilde{p} \\
& \left.+\int_{\tilde{b}_{N}}^{\infty} \mathcal{G}_{P}(\tilde{p})\left(\tilde{p} \sum_{i=1}^{N} q_{i}-\sum_{i=1}^{N} q_{i} c_{i}\right) d \tilde{p}\right) d k
\end{aligned}
$$

The bids that maximize (19) are

$$
\hat{b}_{n}= \begin{cases}c_{n}, & \text { for } n=1 \\ c_{n}+\bar{k} \sum_{i=1}^{n-1} q_{i}, & \text { for } 2 \leq n \leq N\end{cases}
$$

which we derive in the Appendix. $\bar{k}$ is the expectation value of the random variable $K$.

Note that the optimal bids $\hat{b}_{n}$ are independent of the probability distribution of the market price $P$. Therefore, it does not matter what external factors may influence $P$. It may even be subject to strategic action of the competitors.

The optimal bids $\hat{b}_{n}$ represent a step-wise bidding function that is bound to the number of power plants and their respective quantities $q_{i}$. A continuous bidding function is established with the limit $N \rightarrow \infty$. The bids $\hat{b}_{n}$ then change to the bidding function $\hat{b}(q)$, and $c_{n}$ becomes the marginal cost function $c(q)$. With $\sum_{i=1}^{N} q_{i}=Q, q_{i}$ approaches zero, $\sum_{i=1}^{n-1} q_{i}$ approaches $q$, and from (20) follows that

$$
\hat{b}(q)=c(q)+\bar{k} q
$$

\section{B. Optimal Trading Strategy for Integrated Companies}

Integrated companies do not only sell their electricity to the spot market but also to their customers having a fixed contract to a specific price. They have basically two targets when bidding on the spot market: if the spot market price is high enough, they sell electricity and act the same way as a generation-only company would. If the spot market price is smaller than the cost of their own production facilities, they can increase their profit by buying on the spot market instead of producing by themselves. In this case, these companies try to influence the market price by bidding below their cost.

In order to give a formulation for the expected profit, we consider that the $m$ cheapest power plants are needed to satisfy the customers with fixed contracts. We denote the price of the fixed contracts with $a$ and calculate the profit $r$ depending on the influenced market price $\hat{p}$, shown in (22) at the bottom of the next page. The influenced market price $\hat{p}$ is

$$
\hat{p}_{n}= \begin{cases}\tilde{p}, & \text { for } n=1 \\ \tilde{p}-k \sum_{i=1}^{n} \mathrm{~g}\left(c_{i}-\tilde{p}\right) q_{i}, & \text { for } 1<n \leq m \\ \tilde{p}+k \sum_{i=n+1}^{N} \mathrm{~g}\left(\tilde{p}-c_{i}\right) q_{i}, & \text { for } m<n<N \\ \tilde{p}, & \text { for } n=N\end{cases}
$$

which gives for the optimal bids $\hat{b}_{n}$

$$
\hat{b}_{n}= \begin{cases}c_{n}-\bar{k} \sum_{i=n+1}^{m} q_{i}, & \text { for } 1 \leq n<m \\ c_{n}, & \text { for } m \leq n \leq m+1 \\ c_{n}+\bar{k} \sum_{i=m+1}^{n-1} q_{i}, & \text { for } m+1<n \leq N .\end{cases}
$$

From this, we can see that, if demand and supply side are equally powerful, the resulting market price does not change 
very much compared to the case of perfect competition. The resulting market supply function increases by the same amount as the demand function decreases. The equilibrium volume is less than in the competitive case, though.

\section{Influence Factor $k$}

In the previous sections, we found that the influence factor $k$ plays an important role in the decision of the power company. Since $k$ would be useless if we could not determine its value, we derive in the following a relationship between $k$ and the market supply and demand functions.

1) Calculation: Let $l(q, \mathbf{a})$ be the demand function the company faces on the electricity market, which is dependent on the quantity $q$ and the market state a. We denote the slope of this demand function with $k_{l}, k_{l}=(\partial l(q, \mathbf{a})) /(\partial q)$. We further denote the market's demand function with $d(q, \mathbf{a})$ and the supply function resulting from the competing companies with $s(q, \mathbf{a})$. Their slopes taken at the point of market equilibrium are $k_{d}$ and $k_{s}$, respectively.

Since the market supply function results from the sum of the supply functions of the generation companies, the demand function the company faces is equal to market demand less the sum of the supply functions of all competing companies. The sum is taken in volume, so we have to consider the inverse functions of $l(q, \mathbf{a}), d(q, \mathbf{a})$ and $s(q, \mathbf{a})$, denoted by $l^{-1}(p, \mathbf{a}), d^{-1}(p, \mathbf{a})$, and $s^{-1}(p, \mathbf{a})$, for which holds

$$
l^{-1}(p, \mathbf{a})=d^{-1}(p, \mathbf{a})-s^{-1}(p, \mathbf{a})
$$

as illustrated in the left graph of Fig. 2.

The market equilibrium results from the intersection between the bids of the company under consideration and its demand $l(q, \mathbf{a})$. The price difference between influenced and competitive case $\hat{p}-\tilde{p}$ is therefore under linear approximation $\hat{p}-\tilde{p}=$ $-k_{l} \Delta q$, from which follows that $k$ is equal to $-k_{l}$. With (25), we find $\left(1 / k_{l}\right)=\left(1 / k_{d}\right)-\left(1 / k_{s}\right)$ and

$$
k=\frac{k_{s} k_{d}}{k_{d}-k_{s}} .
$$
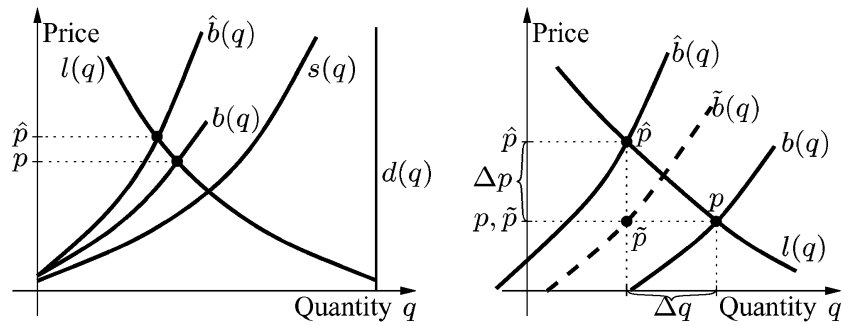

Fig. 2. Comparison of competitive and noncompetitive bidding functions on the electricity market. The area marked by the shaded rectangle in the left graph is shown in more detail in the right graph.

2) Estimation: Econometric estimation of $k_{s}$ and $k_{d}$ therefore allows to derive a value for $k$. In order to get an idea about the value of $k$, we make a very rough estimation on the basis of historic market data from the California electricity market, which is publicly available from the University of California Energy Institute [12]. Since we cannot determine the supply function of specific companies, we estimate $k_{s}$ from the market supply function. The resulting value of $k$ therefore represents the influence of a newly emerging company that does not have its market share yet. We further assume that the demand is perfectly inelastic, so that $k=k_{s}$.

A linear regression of market price on market volume, as shown in Fig. 3, results in a value of $k=k_{s}=$ $2.577 \$ / \mathrm{MWh} / \mathrm{GWh}$. Although this is a very rough estimation that is probably valid for off-peak periods only, the electricity market price shows a noticeable reaction of about 2.6\$/MWh increase for every GWh withheld from the market.

3) Characteristics: In case there is no demand elasticity, $k_{d} \rightarrow-\infty, k$ is equal to the first derivative of the supply function $k=k_{s}$. In case of monopoly, there is no supply function of competing companies $k_{s} \rightarrow \infty$ and $k=-k_{d}$.

The slope of the supply function is in peak periods greater than in off-peak periods. The possible influence on the market price is higher as well, since $k$ increases with increasing slope of the supply function. This results in more market power in peak periods. Therefore, for bidding on multiple auctions, a company will use 24 or 48 different values for $k$, one for each auction.

$$
r(\hat{p})= \begin{cases}\sum_{i=1}^{m} q_{i}(a-\hat{p}), & \text { for } \hat{p}<\hat{b}_{1} \\ \sum_{i=1}^{n} q_{i}\left(a-c_{i}\right)+\sum_{i=n+1}^{m} q_{i}(a-\hat{p}), & \text { for } \hat{b}_{n} \leq \hat{p}<\hat{b}_{n+1} \leq \hat{b}_{m} \\ \sum_{i=1}^{m} q_{i}\left(a-c_{i}\right), & \text { for } \hat{b}_{m} \leq \hat{p}<\hat{b}_{m+1} \\ \sum_{i=1}^{m} q_{i}\left(a-c_{i}\right)+\sum_{i=m+1}^{n} q_{i}\left(\hat{p}-c_{i}\right), & \text { for } \hat{b}_{m+1} \leq \hat{b}_{n} \leq \hat{p}<\hat{b}_{n+1} \\ \sum_{i=1}^{m} q_{i}\left(a-c_{i}\right)+\sum_{i=m+1}^{N} q_{i}\left(\hat{p}-c_{i}\right), & \text { for } \hat{b}_{N} \leq \hat{p}\end{cases}
$$




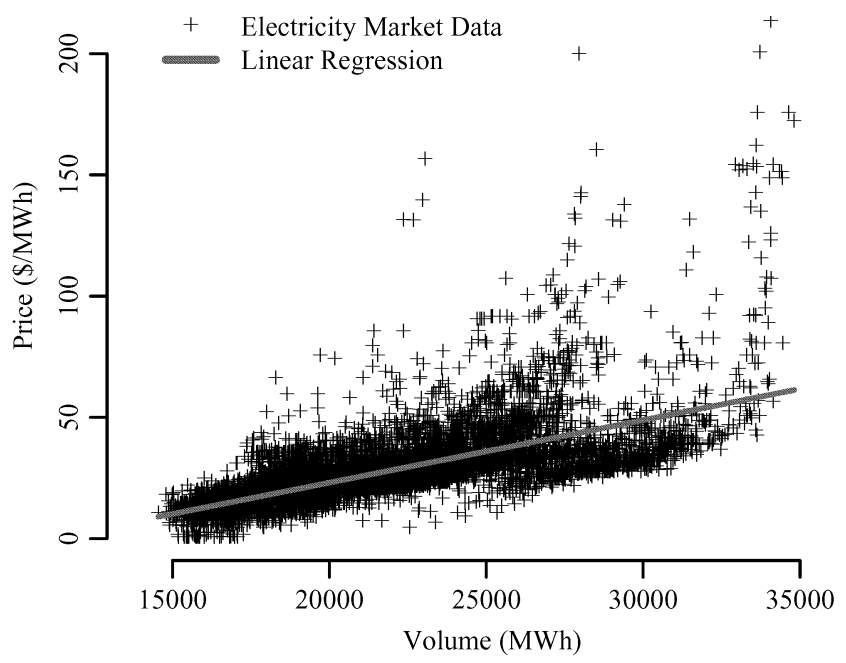

Fig. 3. Linear regression of market price on market volume for the market data of the California electricity market from 1999.

TABLE I

Trading Strategy for a Given Set of POWER Plants

\begin{tabular}{llll}
\hline $\begin{array}{l}\text { Quantity } \\
(\mathrm{MWh})\end{array}$ & $\begin{array}{l}\text { Cost } \\
(\$ / \mathrm{MWh})\end{array}$ & $\begin{array}{l}\text { Calculation } \\
k=2.6 \$ / \mathrm{MWh} / \mathrm{GWh}\end{array}$ & $\begin{array}{l}\text { Bid } \\
(\$ / \mathrm{MWh})\end{array}$ \\
\hline$q_{1}=1000$ & $c_{1}=10$ & $\hat{b}_{1}=c_{1}$ & $\hat{b}_{1}=10$ \\
$q_{2}=1000$ & $c_{2}=11$ & $\hat{b}_{2}=c_{2}+k q_{1}$ & $\hat{b}_{2}=13.6$ \\
$q_{3}=800$ & $c_{3}=15$ & $\hat{b}_{3}=c_{3}+k\left(q_{1}+q_{2}\right)$ & $\hat{b}_{3}=20.2$ \\
$q_{4}=500$ & $c_{4}=20$ & $\hat{b}_{4}=c_{4}+k\left(q_{1}+q_{2}+q_{3}\right)$ & $\hat{b}_{4}=27.28$ \\
\hline
\end{tabular}

$k$ is further influenced by transmission constraints. The electricity exchange handles these constraints by dividing the market into several areas[13]. In times where electricity transmission is insufficient, the market splits into areas with separate market prices and separate values of $k$. If the transmission capacity is sufficient, the connected areas share the market price and their $k$.

$k$ is independent of the marginal cost function of the bidding company. Physical generation constraints like production limits, ramp rates, or minimum up and down times do not influence the value of $k$. The trading strategy is therefore somewhat independent from the generation process of the own company.

\section{Examples}

In order to present a more clear picture of the proposed trading strategy, let us consider a few examples for illustration.

1) Bids for Optimal Influence on the Market Price: We consider a power company with four power plants. The power plants' properties are marginal cost and quantity for one auction, as shown in Table I, columns "Quantity" and "Cost." The calculation of the optimal bids is dependent on the influence factor $k$, which we take from the estimation above. Column "Calculation" shows the details of the calculation, and the resulting optimal bid is given in column "Bid" of Table I. For power plant 4 , we find that due to market power, the bid is about $27 \%$ above marginal cost.

2) Optimal Bid of a Monopolist: For the case of monopoly, the resulting market price can be calculated using the well-known approach of marginal revenue. This allows to
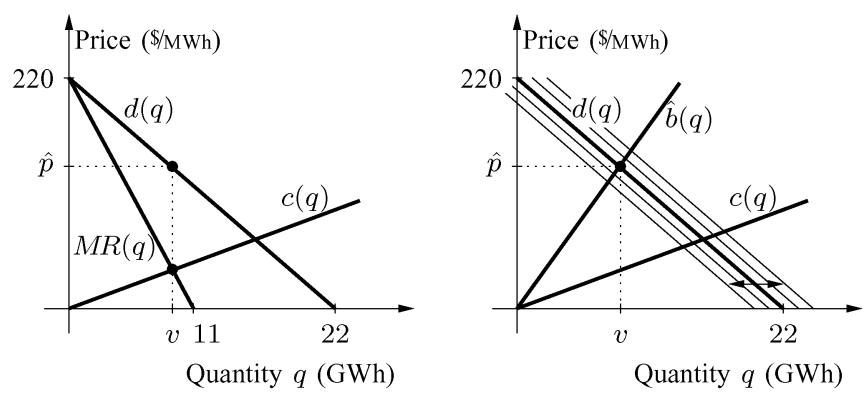

Fig. 4. Calculation of monopoly price by marginal revenue (left graph) and by $k$-factor (right graph).

compare the approach presented in this paper with traditional methods.

For simplicity, let us consider that marginal cost and demand are linear functions in quantity $q$

$$
\begin{array}{ll}
d(q)=k_{d} q+o_{d} & \text { demand } \\
c(q)=k_{c} q+o_{c} & \text { cost. }
\end{array}
$$

$c(q)$ is the marginal cost function, with the slope $k_{c}$ and the offset $o_{c} . o_{d}$ is the demand function's offset. We further consider some values of the functions' parameters, $k_{d}=-10 \quad \$ / \mathrm{MWh} / \mathrm{GWh}, o_{d}=220 \quad \$ / \mathrm{MWh}, k_{c}=$ $2 \$ / \mathrm{MWh} / \mathrm{GWh}$, and $o_{c}=0 \$ / \mathrm{MWh}$.

In the case of the traditional approach, the marginal revenue is then a linear function in volume

$$
\operatorname{MR}(q)=k_{\mathrm{MR}} q+o_{\mathrm{MR}}
$$

with slope $k_{\mathrm{MR}}$ and offset $o_{\mathrm{MR}}$. The marginal revenue is the change of total revenue with changing quantity $\operatorname{MR}(q)=(d T R(q)) /(d q)$, and the total revenue $\operatorname{TR}(q)$ is $\operatorname{TR}(q)=q d(q)$. Therefore

$$
\operatorname{MR}(q)=\frac{d(q d(q))}{d q}=2 k_{d} q+o_{d}
$$

and $o_{\mathrm{MR}}=o_{d}=220 / \$ \mathrm{MWh}$ and $k_{\mathrm{MR}}=2 k_{d}=$ $-20 \$ / \mathrm{MWh} / \mathrm{GWh}$. The market-clearing volume results from the intersection of marginal revenue and marginal cost, i.e., $\operatorname{MR}(v)=c(v) . v$ is the market-clearing volume, which results in $v=10 \mathrm{GWh}$. The corresponding market clearing price $\hat{p}$ is $\hat{p}=120 \$ / \mathrm{MWh}$. The left graph of Fig. 4 illustrates this calculation.

With the method presented in this paper, the market-clearing price can be calculated by construction of the optimal bids of the monopolistic generator. Since the cost are linear, the bidding function $\hat{b}(q)$ is linear as well

$$
\hat{b}(q)=k_{b} q+o_{b}
$$

with slope $k_{b}$ and offset $o_{b}$. With (21) and $k=-k_{d}$, we get $k_{b}=k_{c}-k_{d}$ and $o_{b}=o_{c}=0$. The market equilibrium volume results from the intersection between the bidding curve and the demand function $\hat{b}(v)=d(v)$, which yields for the equilibrium volume $v=10$. As we can see, the results are the same in 
both approaches. To illustrate the difference between these approaches, we compare the calculations in Fig. 4. The left graph shows the conventional approach and the right graph the one presented in this paper. In order to calculate marginal revenue $\operatorname{MR}(q)$, the demand function must be known in detail, i.e., with its offset. In the new approach, the knowledge of $k_{d}$ is sufficient to calculate optimal bids $\hat{b}(q)$. Bidding $\hat{b}(q)$, the market equilibrium will always end up in a perfect monopoly equilibrium, independent of demand shifts, as long as the slope $k_{d}$ is constant. This situation is indicated with thin lines for demand in the right graph of Fig. 4.

3) Oligopoly With Two Players: We consider two companies, $A$ and $B$, both having linear marginal cost functions $c_{A}\left(q_{A}\right)$ and $c_{B}\left(q_{B}\right)$ with equal parameters

$$
\begin{aligned}
& c_{A}\left(q_{A}\right)=k_{s} q_{A}+o_{s} \\
& c_{B}\left(q_{B}\right)=k_{s} q_{B}+o_{s} .
\end{aligned}
$$

The slope $k_{s}$ and the offset $o_{s}$ is common to both companies, the quantities $q_{A}$ and $q_{B}$, the companies get awarded by the electricity market auction, depend on their strategies. We further use the demand function from the last example $d(q)=k_{d} q+o_{d}$.

In the market equilibrium, demand quantity is equal to supply quantity $q=q_{A}+q_{B}$, and the price is

$$
p=k_{d}\left(q_{A}+q_{B}\right)+o_{d} .
$$

The respective profits $r_{A}$ and $r_{B}$ are total revenue less total cost

$$
\begin{aligned}
r_{A} & =p q_{A}-o_{A} q_{A}-\frac{1}{2} k_{s} q_{A}^{2} \\
& =q_{A}^{2}\left(k_{d}-\frac{k_{s}}{2}\right)+q_{A}\left(k_{d} q_{B}+o_{d}-o_{s}\right) \\
r_{B} & =p q_{B}-o_{B} q_{B}-\frac{1}{2} k_{s} q_{B}^{2} \\
& =q_{B}^{2}\left(k_{d}-\frac{k_{s}}{2}\right)+q_{B}\left(k_{d} q_{A}+o_{d}-o_{s}\right) .
\end{aligned}
$$

a) Conventional oligopoly approach: We calculate the optimal bids according to an oligopolistic Cournot model and use the quantities as decision variables. This approach is used by the majority of research work on oligopolistic electricity markets, e.g., [14] analyzes electricity market price peaks in a similar setting like in this example. References [4] and [15] investigate more complex electricity market configurations. A Nash equilibrium is reached, if both companies cannot increase their profits by adapting their offers. Therefore, the first-order condition $\left(d r_{A}\right) /\left(d q_{A}\right)=0$ and $\left(d r_{B}\right) /\left(d q_{B}\right)=0$ must be met, and

$$
\begin{aligned}
& q_{A}\left(2 k_{d}-k_{s}\right)+k_{d} q_{B}+o_{d}-o_{s}=0 \\
& q_{B}\left(2 k_{d}-k_{s}\right)+k_{d} q_{A}+o_{d}-o_{s}=0,
\end{aligned}
$$

which results in

$$
\begin{aligned}
q_{A}=q_{B} & =-\frac{o_{s}-o_{d}}{k_{s}-3 k_{d}} \\
p & =o_{d}-\frac{2 k_{d}\left(o_{s}-o_{d}\right)}{k_{s}-3 k_{d}} \\
r_{A}=r_{B} & =\frac{\left(k_{s}-2 k_{d}\right)\left(o_{s}-o_{d}\right)^{2}}{2\left(k_{s}-3 k_{d}\right)^{2}} .
\end{aligned}
$$

b) k-Factorfor the Cournot model: The basic assumption in a Cournot model is that every company considers the competitors placing bids with a constant quantity. Therefore, the slope of the competitors' bidding functions is considered infinite and $k=$ $-k_{d}$. Under this assumption, the optimal bidding function is

$$
\hat{b}\left(q_{s}\right)=\left(k_{s}-k_{d}\right) q_{s}+o_{s}
$$

$q_{s}$ denotes the quantity supplied by each company, $q_{s}=q_{A}=$ $q_{B}$. The market equilibrium is found at $q=2 q_{s}$

$$
2 k_{d} q_{s}+o_{d}=\left(k_{s}-k_{d}\right) q_{s}+o_{s}
$$

which is equivalent to (34) and gives therefore the same results (35) as the conventional approach. The main difference is that the companies bid, in contrary to their own assumptions, with a finite slope $\hat{k}_{A}=\hat{k}_{B}=k_{s}-k_{d}$, which is not an optimal strategy, as we show in the following.

Let us consider both companies bidding with different strategies. $B$ decides to use the Cournot approach and bids with $\hat{k}_{B}=$ $k_{s}-k_{d}$. A knows $B$ 's strategy and uses $\hat{k}_{A}=k_{s}+k$ and

$$
k=\frac{\hat{k}_{B} k_{d}}{k_{d}-\hat{k}_{B}}=\frac{k_{s} k_{d}-k_{d}^{2}}{2 k_{d}-k_{s}} .
$$

In the market equilibrium, the quantities the companies get awarded are

$$
q_{B}=\frac{o_{d}-o_{s}}{2\left(k_{s}-2 k_{d}\right)}, \quad q_{A}=\frac{o_{d}-o_{s}}{-2 k_{d}} .
$$

Obviously, company $B$ has less volume than $A$ and therefore less profit, since price and marginal cost function are common to both of them.

So we must conclude from this example that all companies must bid with a predefined volume in order to fulfill the assumption of constant quantity for their competitors. This strategy circumvents the market mechanisms of the auction market and its capability of creating a market equilibrium in price and volume, since the volume is decided in advance. From the viewpoint of the auction market, the companies divide the market among them. Therefore, the Cournot model represents a collusive market equilibrium and would give unreasonably high equilibrium prices for a low demand elasticity, as discussed in [16].

c) Optimal trading strategy: With (21) and (26), the optimal bidding function is for both companies $\hat{b}\left(q_{s}\right)=\hat{k}_{s} q_{s}+o_{s}$, and the cost functions are the same as before. $\hat{k}_{s}$ is the optimal slope of the bidding function for maximum expected profit. It is dependent on the slope of the competing company, which is $\hat{k}_{s}$ as well and therefore

$$
\hat{k}_{s}=k_{s}+\frac{k_{d} \hat{k}_{s}}{k_{d}-\hat{k}_{s}}
$$

and $\hat{k}_{s}=(1 / 2)\left(\sqrt{k_{s}^{2}-4 k_{s} k_{d}}+k_{s}\right)$. 
Using a conventional approach, the equilibrium point for strategies with arbitrary supply functions is found with the supply function equilibrium [17]

$$
S^{\prime}(\breve{p})=\frac{S(\breve{p})}{\breve{p}-C^{\prime}(S(\breve{p}))}+D^{\prime}(\breve{p}) .
$$

$C(q)$ is the total cost function, $S(\breve{p})$ is the supply function, and $D(\breve{p})$ is the demand function. Equation (41) is defined for $C^{\prime}(0)=0$, so $\breve{p}$ represents a price relative to the offset of the marginal cost function, $\breve{p}=p-o_{s}$, and $C^{\prime}(q)=c(q)-o_{s}=$ $k_{s} q$. Demand and supply are functions of the price, $S(\breve{p})=$ $\hat{b}^{-1}(\breve{p})=\left(1 / \hat{k}_{s}\right) \breve{p}$, and $D(\breve{p})=d^{-1}(\breve{p})=\left(1 / k_{d}\right) \breve{p}$. Their first derivatives are $S^{\prime}(\breve{p})=1 / \hat{k}_{s}$ and $D^{\prime}(\breve{p})=\left(1 / k_{d}\right)$, and therefore

$$
\frac{1}{\hat{k}_{s}}=\frac{\frac{1}{\hat{k}_{s}} \breve{p}}{\breve{p}-\frac{k_{s}}{\hat{k}_{s}} \breve{p}}+\frac{1}{k_{d}}
$$

which can be rewritten to (40).

The examples show that the approach presented in this paper is equivalent to conventional methods like Cournot-Nash equilibrium or supply function equilibrium, if their respective assumptions are added.

\section{Market Design Analysis}

The previous sections show that it is very easy for the companies to increase their profits at low risk by bidding higher than marginal cost. There is little reason why companies should bid less than the price that maximizes their profits. Therefore, the duty of market design is to prohibit strategic bidding by means of an adequate set of market rules. In particular, since we know the point of maximum profit of a company (21), we can compare the result with ideal conditions and check possible market rules for their effectiveness in making the market ideal competitive.

\section{A. Changing Auction Format to Pay-as-Bid Auctions}

If all market participants use the trading strategy of maximum profit (9), the resulting market price is higher than the marginal cost. Furthermore, when considering repeated auctions over time starting at marginal cost, the resulting market price is higher than marginal cost after the first trading step. This increases the expected market price, which in turn increases the optimal bids on the following trading day. This means that market prices may rise, independent of number and size of market participants, until an upper bound is reached, which must be given by law.

The optimal trading strategy for pay-as-bid auctions is derived under the assumption of perfect competition, i.e., a single company does not have influence on the market price. Therefore, the effect of rising market prices may even be worse if the company's influence is considered.

This auction format allows for bids above marginal cost, even in the competitive case. Furthermore, the optimal bid rises with the market price expectation, which makes the market-clearing price unstable. Therefore, this auction format is with respect to the possibility of gaming inferior to the uniform-price auction.

\section{B. Increasing the Number of Market Participants}

Besides changing the auction format, another prominent action in increasing the competition on the electricity market is to increase the number of market participants. This can be done by dividing existing companies or by decreasing the barriers for new companies entering the market. Though it is obvious that a higher number of companies will increase competition, we can estimate the effectiveness of such a change in the market.

With (21), the deviation of the bidding function from marginal cost $\hat{b}(q)-c(q)$ is proportional to the volume $q$. In a scenario of $M$ equally sized companies being divided into $2 M$ equally sized smaller companies, the companies' influence on the market price will be cut in half, since their volume $q$ decreases correspondingly. In a scenario of some small companies entering a market of well-established big companies, the influence of those big companies will not change very much.

Although a higher number of market participant can decrease market power, there will always remain a significant amount that cannot be removed. So this approach is not sufficient for perfect competition.

\section{Demand-Side Management}

The market power of the companies strongly depends on the expected value of $k$, which in turn depends on the slope of demand and supply function (26). For a closer investigation in the influence of demand-side management, we define $k_{d}$ relative to $k_{s}, k_{d}=-h k_{s}, h \geq 0$. The influence factor $k$ then becomes

$$
k=k_{s} \frac{h}{h+1}
$$

from which we can see that, if the demand side is quite inelastic compared to the supply side, e.g., $h=20$, then a very big change on the demand side to $h=10$ will not have very much influence on $k: k=0.95 k_{s}$ for $h=20$ changes to $k=0.91 k_{s}$ with $h=10$. Therefore, demand-side management will be a drop in the ocean unless we manage to make the demand side as elastic as the supply side, i.e., $h=1$ or $k_{d}=-k_{s}$, which means that there are full generation companies with excess capacity on the demand side.

\section{Price Caps}

From the viewpoint of a generation company, the characteristics of the market, i.e., the slope of demand and supply, do not change with the introduction of price caps. Therefore, the optimal trading strategy will not change for bids below the price cap.

If the optimal bid would exceed the price cap, the company basically has two options: first, bid with a price equal to the price cap. This strategy will result in some difficulties for the operator of the energy exchange, who must decide how to distribute the equilibrium volume to suppliers with equal bids, in case the market equilibrium reaches the price cap. The second option for the supplying company is to truncate its bidding function at the price cap. This will probably be the preferred strategy, since the market will not clear if demand exceeds supply at the price cap, and the system operator would be forced to remove the price cap in order to ensure reliable electricity supply. 
Therefore, price caps may lead to reliability problems, as discussed in [8], or their introduction fails, as explained for the California electricity market by Wilson [7, Appendix], who says "One lesson learned was that a price cap is meaningless unless the System Operator curtails demand when supplies offered at the price cap are insufficient."

\section{E. Summary}

With (21), we find that big companies can influence the market price more easily than small ones. This corresponds to common market understanding and proves the presumption of Borenstein [1] that small companies may also have influence. It also matches the quantitative analysis of Bialek [18], who found that the market power may be proportional to the "effective market share," and the arguments of Bunn and Martoccia [19], who state that it is clear that "a generator would generally mark-up plant higher in the stack proportionally more than those below it."

Together with the theoretical viewpoint presented in this paper, these findings can be summarized with the following proposition.

Proposition 1: Considering a uniform-price electricity auction market and $M$ participants, each of them able to influence the market price only by adapting their supply functions, their optimal bids are equal to marginal cost if and only if one of the following conditions is satisfied:

1) the expected slope of the cumulative supply function of any $M-1$ participants is zero, $k_{s}=0$;

2) the expected slope of the demand function is equal to zero, $k_{d}=0$.

Proof: The optimal bidding function $b(q)$ is, under linear approximation in the point of perfect competition (21), $b(q)=$ $c(q)+\bar{k} q$. Because the approximation is exact in the point of perfect competition, it follows that $b_{n}=c_{n}$ is fulfilled for every company if and only if $\bar{k}$ is equal to zero. With (26) and (25), this is true for any of the $M$ participants if the slope of the cumulative supply function of the remaining $M-1$ companies or the slope of the demand function are known to be zero, $k_{s}=0$, or $k_{d}=0$.

Although an electricity auction market suffers from market power if none of these conditions is fulfilled, it seems little practicable for deriving recommendations for real markets, since the slopes of demand and supply function will always differ from zero. We must therefore follow that an electricity market can never be perfectly competitive.

However, if we consider any real electricity auction market and denote the number of generation companies with $M$, then it is almost certain that we find at least one company such that the remaining $M-1$ companies are not able to satisfy the whole market's demand. The $M$ th company can bid at any high price without reaching a point of zero demand, it enjoys a situation of "unrestricted market power."

Therefore, it must be required that any $M-1$ companies have enough generation capacity to satisfy the whole market demand in order to prevent from "unrestricted market power." This finding corresponds to the arguments of Haas [20], who states that excess capacity is a necessary requirement for a functioning electricity market. The above relation can be summarized with another, less restrictive proposition.

Proposition 2: An electricity market with $M$ participants is free of "unrestricted market power" if and only if any $M-1$ participants are able to satisfy the whole market demand.

\section{CONCLUSION}

Once we accept that the electricity market is noncompetitive with all its consequences, namely, that the market participants do have influence on the market price, we find a view on the electricity market that combines the perceptions and observations of recent research into one picture that answers open questions about market power on the electricity market.

The existence of market power follows directly from the single assumption of noncompetitiveness. Oligopoly assumptions may be added to find the point of maximum market influence, and econometric methods may be used to evaluate the actual conditions of a specific market.

We show in this paper an optimal trading strategy for generation companies under uncertainty, which allows us, apart from well-known approaches like Cournot-Nash games or assumptions of collusion, to explain the companies' behavior, resulting in market power and a high market price volatility. This trading strategy is independent of traditional assumptions used for modeling the market under consideration. However, adapting the parameter $k$ according to these assumptions makes the method presented in this paper equivalent to conventional approaches, which we explicitly show for the case of monopoly, Cournot game, supply function equilibrium, and perfect competition.

On the basis of this trading strategy, we derive necessary and sufficient conditions for establishing an ideal competitive electricity auction market. Unfortunately, these conditions are far from being realizable, which makes us conclude that an electricity auction market can never be perfectly competitive in a deregulated environment.

From this viewpoint, it was an illusion to believe that a higher number of supplying companies is sufficient to eventually bring a competitive market price. Unconditional deregulation seems to end up in a situation of survival of the fittest, which, in contrast to common economic belief, does not lead to perfect competition in the sense that the market price is equal to marginal cost. It rather equips the fittest with the necessary instruments to extend its dominant position. Perfect competition therefore does not emerge on its own; it needs to be introduced by a carefully designed market environment.

APPENDIX

Optimal Bid on a UNIFORM-PRICE AUCTION MARKET

\section{A. In a Perfect Competitive Market}

The first-order condition $(\partial \bar{r}) /\left(\partial b_{n}\right)=0$ for (12) is

$$
-\mathcal{G}_{P}\left(b_{1}\right)\left(b_{1} q_{1}-q_{1} c_{1}\right)=0
$$


for $n=1$ and

$$
\begin{aligned}
& \mathcal{G}_{P}\left(b_{n}\right)\left(b_{n} \sum_{i=1}^{n-1} q_{i}-\sum_{i=1}^{n-1} q_{i} c_{i}\right) \\
& -\mathcal{G}_{P}\left(b_{n}\right)\left(b_{n} \sum_{i=1}^{n} q_{i}-\sum_{i=1}^{n} q_{i} c_{i}\right)=0
\end{aligned}
$$

for $1<n \leq N$. Both cases yield after collection of summands

$$
\mathcal{G}_{P}\left(b_{n}\right) q_{n}\left(c_{n}-b_{n}\right)=0 .
$$

Since $q_{n} \neq 0$, possible solutions are $\mathcal{G}_{P}\left(b_{n}\right)=0$ and $b_{n}=$ $c_{n} \cdot\left(\partial^{2} \bar{r}\right) /\left(\partial b_{n}^{2}\right)$ gives information about the quality of these solutions

$$
\frac{\partial^{2} \bar{r}}{\partial b_{n}^{2}}=\mathcal{G}_{P}^{\prime}\left(b_{n}\right) q_{n}\left(c_{n}-b_{n}\right)-\mathcal{G}_{P}\left(b_{n}\right) q_{n} .
$$

For $\mathcal{G}_{P}\left(b_{n}\right)=0$, we find that $\mathcal{G}_{P}^{\prime}\left(b_{n}\right)=0$ as well, since $\mathcal{G}_{P}(x) \geq 0 \forall x$. Furthermore, the derivatives left and right of $b_{n}$ are of opposite sign, again because $\mathcal{G}_{P}\left(b_{n}\right)=0$ and $\mathcal{G}_{P}(x) \geq$ $0 \forall x$. Therefore, for $\mathcal{G}_{P}\left(b_{n}\right)=0$, we get $\left(\partial^{2} \bar{r}\right) /\left(\partial b_{n}^{2}\right)=0$, representing a saddle point, which is not a valid maximum. ${ }^{2}$ For $b_{n}=c_{n},\left(\partial^{2} \bar{r}\right) /\left(\partial b_{n}^{2}\right)=-\mathcal{G}_{P}\left(b_{n}\right) q_{n}<0$, and therefore, $b_{n}=c_{n}$ is a valid maximum

\section{B. Without Assumption of Perfect Competition}

Applying $(\partial \bar{r}) /\left(\partial \tilde{b}_{n}\right)=0$ on (19) gives for $1<n<N$

$$
\begin{aligned}
\int_{-\infty}^{\infty} \mathcal{G}_{K}(k)\left(\mathcal{G}_{P}\left(\tilde{b}_{n}\right)\right. & \left(\left(\tilde{b}_{n}+k \sum_{i=n}^{N} \mathrm{~g}\left(\tilde{b}_{n}-c_{i}\right) q_{i}\right)\right. \\
\times & \left.\sum_{i=1}^{n-1} q_{i}-\sum_{i=1}^{n-1} q_{i} c_{i}\right) \\
- & \mathcal{G}_{P}\left(\tilde{b}_{n}\right)\left(\left(\tilde{b}_{n}+k \sum_{i=n+1}^{N} \mathrm{~g}\left(\tilde{b}_{n}-c_{i}\right) q_{i}\right)\right. \\
& \left.\left.\times \sum_{i=1}^{n} q_{i}-\sum_{i=1}^{n} q_{i} c_{i}\right)\right) d k=0
\end{aligned}
$$

and further

$$
\begin{aligned}
0= & \int_{-\infty}^{\infty} \mathcal{G}_{K}(k)\left(-\tilde{b}_{n} q_{n}+\left(k \sum_{i=n}^{N} \mathrm{~g}\left(\tilde{b}_{n}-c_{i}\right) q_{i}\right)\right. \\
& \times \sum_{i=1}^{n-1} q_{i}-\left(k \sum_{i=n+1}^{N} \mathrm{~g}\left(\tilde{b}_{n}-c_{i}\right) q_{i}\right) \\
& \left.\times \sum_{i=1}^{n} q_{i}+q_{n} c_{n}\right) d k .
\end{aligned}
$$

${ }^{2}$ For non-differentiable $\mathcal{G}_{P}\left(b_{n}\right)$, we may replace the derivatives with the corresponding differential quotients to show the quality of the solution $\mathcal{G}_{P}\left(b_{n}\right)=$ 0 .
Collection of the summands yields

$$
\begin{aligned}
0=\int_{-\infty}^{\infty} & \mathcal{G}_{K}(k)\left(k \mathrm{~g}\left(\tilde{b}_{n}-c_{n}\right) q_{n} \sum_{i=1}^{n-1} q_{i}\right. \\
& \left.-k \sum_{i=n+1}^{N} \mathrm{~g}\left(\tilde{b}_{n}-c_{i}\right) q_{i} q_{n}-\tilde{b}_{n} q_{n}+q_{n} c_{n}\right) d k
\end{aligned}
$$

After elimination of $q_{n}$ and with $\mathrm{g}\left(\tilde{b}_{n}-c_{n}\right)=1$ follows

$$
\begin{aligned}
0=\int_{-\infty}^{\infty} \mathcal{G}_{K}(k)\left(c_{n}\right. & +k \sum_{i=1}^{n-1} q_{i} \\
& \left.\quad \tilde{b}_{n}-k \sum_{i=n+1}^{N} \mathrm{~g}\left(\tilde{b}_{n}-c_{i}\right) q_{i}\right) d k
\end{aligned}
$$

which, using (16) and $\int_{-\infty}^{\infty} \mathcal{G}_{K}(k) k d k=\bar{k}$, yields (20).

In case $n=1$, from $(\partial \bar{r}) /\left(\partial \tilde{b}_{1}\right)=0$ follows

$$
\begin{aligned}
0=\int_{-\infty}^{\infty} & \mathcal{G}_{K}(k) \mathcal{G}_{P}\left(\tilde{b}_{1}\right) \\
& \times\left(\left(\tilde{b}_{1}+k \sum_{i=2}^{N} \mathrm{~g}\left(\tilde{b}_{1}-c_{i}\right) q_{i}\right) q_{1}-q_{1} c_{1}\right) d k
\end{aligned}
$$

which leads with elimination of $\mathcal{G}_{P}(\tilde{p})$ and $q_{1}$ (16) and $\int_{-\infty}^{\infty} \mathcal{G}_{K}(k) d k=1$ to $(20)$.

$$
\text { For } n=N,(\partial \bar{r}) /\left(\partial \tilde{b}_{N}\right)=0 \text { gives }
$$

$$
\begin{aligned}
0= & \int_{-\infty}^{\infty} \mathcal{G}_{K}(k)\left(\mathcal { G } _ { P } ( \tilde { b } _ { N } ) \left(\left(\tilde{b}_{N}+k \mathrm{~g}\left(\tilde{b}_{N}-c_{N}\right) q_{N}\right)\right.\right. \\
& \left.\times \sum_{i=1}^{N-1} q_{i}-\sum_{i=1}^{N-1} q_{i} c_{i}\right) \\
& \left.-\mathcal{G}_{P}\left(\tilde{b}_{N}\right)\left(\tilde{b}_{N} \sum_{i=1}^{N} q_{i}-\sum_{i=1}^{N} q_{i} c_{i}\right)\right) d k \\
= & \int_{-\infty}^{\infty} \mathcal{G}_{K}(k)\left(k q_{N} \sum_{i=1}^{N-1} q_{i}-\tilde{b}_{N} q_{N}+q_{N} c_{N}\right) d k
\end{aligned}
$$

from which follows again (20).

\section{ACKNOWLEDGMENT}

The authors would like to thank the anonymous reviewers for the comments, which led to the deeper insight that "market power" may also include the power to change legal restrictions.

\section{REFERENCES}

[1] S. Borenstein, "The trouble with electricity markets: Understanding California's restructuring disaster," J. Econ. Perspect., vol. 16, no. 1, pp. 191-211, 2002.

[2] S. Hao, "A study of basic bidding strategy in clearing pricing auctions," IEEE Trans. Power Syst., vol. 15, no. 3, pp. 975-980, Aug. 2000.

[3] R. Green, "Increasing competition in the British electricity spot market," J. Ind. Econ., vol. 44, no. 2, pp. 205-216, Jun. 1996.

[4] A. R. Kian, J. B. Cruz, Jr., and R. J. Thomas, "Bidding strategies in oligopolistic dynamic electricity double-sided auctions," IEEE Trans. Power Syst., vol. 20, no. 1, pp. 50-58, Feb. 2005. 
[5] Y. Ren and F. D. Galiana, "Pay-as-bid versus marginal pricing-Part I: Strategic generator offers," IEEE Trans. Power Syst., vol. 19, no. 4, pp. 1771-1776, Nov. 2004.

[6] — , "Pay-as-bid versus marginal pricing-Part II: Market behavior under strategic generator offers," IEEE Trans. Power Syst., vol. 19, no. 4, pp. 1777-1783, Nov. 2004.

[7] R. Wilson, "Architecture of power markets," Econometrica, vol. 70, no. 4, pp. 1299-1340, Jul. 2002.

[8] P. L. Joskow and J. Tirole, Reliability and competitive electricity markets National Bureau of Economic Research, Inc., May 2004, NBER Working Papers 10472.

[9] E. Rasmusen, Games and Information. Malden, MA: Blackwell, 1996.

[10] V. P. Gountis and A. G. Bakirtzis, "Bidding strategies for electricity producers in a competitive electricity marketplace," IEEE Trans. Power Syst., vol. 19, no. 1, pp. 356-365, Feb. 2004

[11] T. Li and M. Shahidehpour, "Strategic bidding of transmission-constrained GENCOs with incomplete information," IEEE Trans. Power Syst., vol. 20, no. 1, pp. 437-447, Feb. 2005.

[12] California Electricity Market Data The University of California Energy Institute, 2003 [Online]. Available: http://www.ucei.berkeley.edu/ CSEM/datamine/datamine.htm.

[13] EEX Spot Market Concept European Energy Exchange EEX, Aug. 2005, Tech. Rep. [Online]. Available: http://www.eex.de/info_center/ downloads/dl_spot/booklet_e.pdf.

[14] X. Guan, Y.-C. Ho, and D. L. Pepyne, "Gaming and price spikes in electric power markets," IEEE Trans. Power Syst., vol. 16, no. 3, pp. 402-408, Aug. 2001.

[15] V. P. Gountis and A. G. Bakirtzis, "Efficient determination of Cournot equilibria in electricity markets," IEEE Trans. Power Syst., vol. 19, no. 4, pp. 1837-1844, Nov.. 2004.
[16] C. J. Day, B. F. Hobbs, and J.-S. Pang, "Oligopolistic competition in power networks: A conjectured supply function approach," IEEE Trans. Power Syst., vol. 17, no. 3, pp. 597-607, Aug. 2002.

[17] P. D. Klemperer and M. A. Meyer, "Supply function equilibria in oligopoly under uncertainty," Econometrica, vol. 57, no. 6, pp. 1243-1277, 1989.

[18] J. Bialek, "Gaming the uniform-price spot market: Quantitative analysis," IEEE Trans. Power Syst., vol. 17, no. 3, pp. 768-773, Aug. 2002.

[19] D. W. Bunn and M. Martoccia, "Unilateral and collusive market power in the electricity pool of England and Wales," Energy Econ., vol. 27, pp. 305-315, 2005.

[20] R. Haas, "The role of excess capacities for effective competition in electricity markets," in Proc. 6th IAEE Eur. Conf., Sep. 2004

Walter Reinisch received the Dipl.Ing. degree in electric and electronic engineering from Vienna University of Technology, Vienna, Austria, in 1997. He is currently pursuing the Ph.D. degree at the Graduate School of Energy Science, Kyoto University, Kyoto, Japan.

He was with SIEMENS AG Österreich, Program and System Engineering Department in the field of power system operation control until October 2003.

Tetsuo Tezuka received the Dr.Eng. degree from the University of Tokyo, Tokyo, Japan, in 1983.

After that, he was an Assistant with the Department of Electric Engineering, Kyoto University, Kyoto, Japan. From May 1996 until April 2003, he was an Associate Professor at the Graduate School of Energy Science, Kyoto University, where he presently is a Professor. 